\title{
Design support methodology using quality function deployment with feature-based reasoning
}

\author{
Zeyad M. El-Kahlout, C. S. Irgens, Barbara M. Savage \\ Faculty of Engineering, The University of the West of England \\ Bristol, Coldharbour Lane, Bristol BS16 1QY.
}

\begin{abstract}
This paper reviews the benefits and short comings of Quality Function Deployment as a design support tool. It identifies the principal problem areas, considers briefly work which has already been done to address them, and then focusses on a proposed solution for one of these problem areas. It is suggested that a FeatureBased Reasoning approach would be a beneficial addition to the evaluation process to determine the relation between the WHATs and the HOWs, and that this would result in an improvement in the ease of use and robustness of this part of the QFD process.
\end{abstract}

\section{Keywords}

Quality Function Deployment, Design, Feature-based Reasoning

\section{INTRODUCTION}

Quality Function Deployment (QFD), is a concept which Professor Yoji Akao introduced in Japan back in 1966. He defined QFD as follows; "QFD is converting the consumers' demands into 'quality characteristics' and developing a design quality for the finished product by systematically deploying the relation between the demands and the characteristics, starting with the quality of each functional component and extending the deployment to the quality of each part and process." Zairi(1995)

Although QFD implementation in design of a new product leads to measurable benefits, users of QFD still encounter a number of serious problems. The purpose of this paper is to review these problems and conduct a preliminary analysis in 
order to define the problem and consequently give some hypothetical solutions, which will be the start point in the primary author's Ph.D. research work.

\section{BENEFITS OF QFD}

A number of potential benefits may acrue from using QFD in design of a new product. In summary, these are:-

- QFD helps define product specifications to meet the customer's requirements, while paying attention to the competitors.

- QFD helps in ensuring consistency between the customer's requirements and the measurable characteristics of the product.

- QFD ensures consistency between the planning and the production process.

- QFD decreases time and cost because planning takes place at an earlier stage .

\section{SHORTCOMINGS OF QFD}

Some major short comings have been reported by a number of well known organisations and/or researchers (Zairi(1995), Hamza(1996)) when using the QFD technique in design of a new product. These are:-

- Mixed consumer needs; mandatory needs plus optional requirements;

- Long learning curve;

- Lack of reliable objective input in the right form;

- Too focused on the mechanics of scoring;

- High subjectivity in the evaluation processes;

- High dependance upon the availability and the level of experience in the team carrying out the work; thus leading to problems in marshalling corporate expertise and experience.

\section{PRELIMINARY ANALYSIS}

Study of the above mentioned problems makes it clear that they lie in several different parts of the QFD process (as indicated in figure 1). Theses problems can be seen to take the form of several queries which are stated below. 


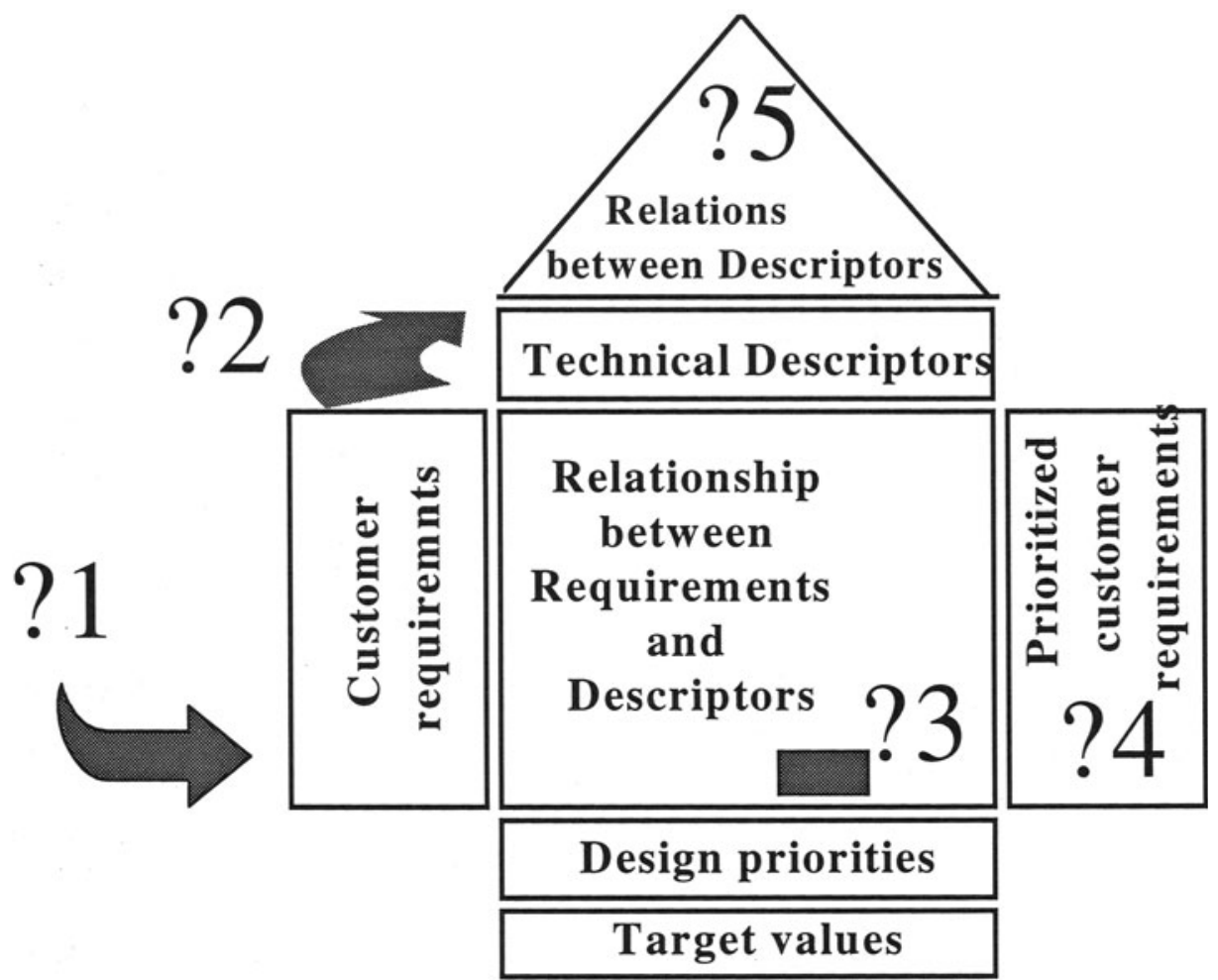

Figure 1 The areas of the major problems in QFD

- Query 1: How to arrange the data in the proper pattern

- Query 2: How these requirements are translated into Technical descriptors

- Query 3: How to evaluate and score the relation between the descriptors and the requirements

- Query 4: How to prioritise the customer requirements

- Query 5: How to determine and evaluate the interrelations between the descriptors

\section{DEFINITION OF THE PROBLEM}

It has been realised by researchers, (e.g. Irgens(1990) and Hamza(1996)) that the root problem behind these shortcomings of QFD may be the high degree of subjectivity of evaluation for both the customer requirements and the technical descriptors. The effects of this degree of subjectivity can be directly related to the level of experience and knowledge of the team undertaking the QFD analysis. 
Thus it is desireable that customer requirements and associated design solutions should be determined using more objective and experiential information.

Several researchers (e.g.Irgens (1990), Adams (1997) and Hamza (1996)) have attempted to solve this problem by using supporting techniques in conjunction with the QFD. For example, the use of Case-Based Reasoning (CBR), in the stage of translating the customer requirements into technical descriptors i.e. the planning stage. This has met with some success and has improved the situation and provided a useful tool.

Another step forward was made by developing a system whereby the QFD engineer may receive advice and assistance at any stage of the QFD process based on previous experience from all stages and stored in a database (Adams (1997)). However, all these major improvements to QFD have only dealt with the WHATs and HOWs, which are the customer requirements and the technical descriptors matrices respectively, and the translation process between them.

The area that has not been dealt with yet is the area marked in figure 1 as "? 3 ", which is the relation between the WHATs and the HOWs and the evaluation of this relation, currently determined using a simple scoring system.

\section{THE PROPOSED WORK}

The proposed solution for the problem identified above is to use a reasoning approach in the evaluation process of the relation between the WHATs and the HOWs. This proposed reasoning approach will be based around the concept of feature modelling.

Human problem solving tends to be based on the identification and processing of the problem features. This uses stored knowledge and experience which is also organised in a feature-based manner. It is believed that we reason by analogy, adapting known models to the current problem situation (Shah (1995)).

These known models, built from a set of accumulated knowledge, contain the features that are to be used to construct the problem solution, for instance, the new product.

Referring to the QFD process introduced in Adams(1997), it can be seen that in the early stages of design it is important to set the design specifications (the HOWMUCH, or the target values) as close as possible to the customer requirements (see figure 2 below). This should reduce the amount of work required in the subsequent stages, thus reducing the total number of iterations 
required to meet the customer requirements. This is the actual intention of the QFD process.

The term Feature usually implies the generic shapes or characteristics of a product with which engineers can associate certain knowledge and attributes, used for reasoning about the product (Shah(1995)), thus features could be divided into form features, functional features, etc.

Feature-based Knowledge encapsulates a full definition of the product geometry and function. We can view features as modelling entities that allow commonly used shapes to be characterised and associated with a set of attributes relevant to a given product type or family.

This approach is dependent upon a suitable product model so that the desired functions of the product can be correlated with its geometric attributes. A system philosophy formulated for this purpose is described in Irgens(1990). This approach was successfully used to generate a set of tree and graph structures indicating the relationships between the various features of which the product was composed.

The product functionality was decomposed successively into function components and weighted so that an overall measure of functional capability could be computed at any level of decomposition. Product quality was seen as the ability to satisfy the customer requirements specification and as such would conform to the QFD approach. This was done by at the product design stage by selecting a suitable set of form features as the carriers for the design intent. Each form feature would contribute to the product's overall, emerging properties, in combination with other form features. The knowledge about the individual form feature's contribution was connected to the set of desired product function components by a set of rule-bases. These rule-bases were based upon the accumulated knowledge. This knowledge handles the interpretation of past customer requirements specification (CRS) and associated design solutions which produced a known functional behaviour. These rule bases allowed reasoning about the likely outcome of the product's functional behaviour, once the desired CRS and type of product was known.

It is the authors' intention to demonstrate that QFD can be beneficially supported by the use of Feature-Based Reasoning. In this way it should be possible to make the QFD process more responsive to accumulated expertise, and possibly more accurately reflecting this in the design of new product variants. 


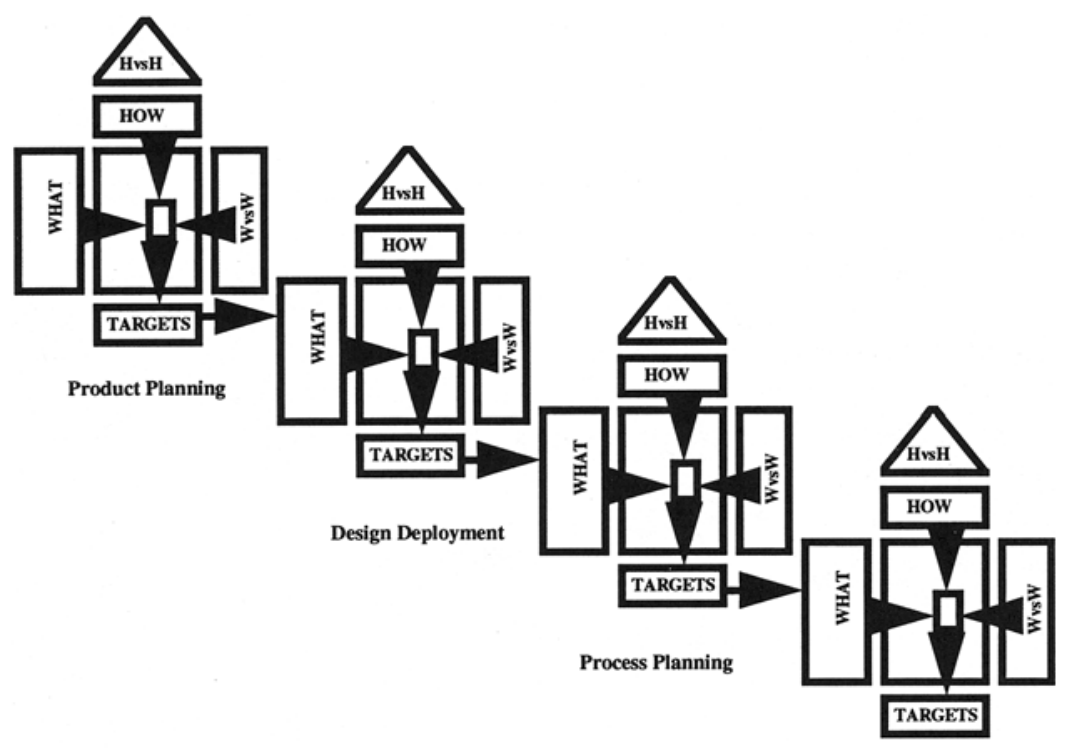

Production Planning

Figure 2 The QFD Process (Adams(1997))

In the proposed methodology it is the authors' intention to try to establish a reasoning shell which will be fed with the CRS (WHATs) and the technical descriptors (HOWs) which have been translated from the customer requirement using Case-based Reasoning (CBR). Then the designed shell will use the FeatureBased Reasoning (FBR) to evaluate the relation between the WHATs and the HOWs and, once this has been established, it will be much easier to obtain the WHATs vs WHATs, WHATs vs HOWs, HOWs vs HOWs, the HOWMUCH values and relations with a high degree of objectivity and accuracy (see figure3). 


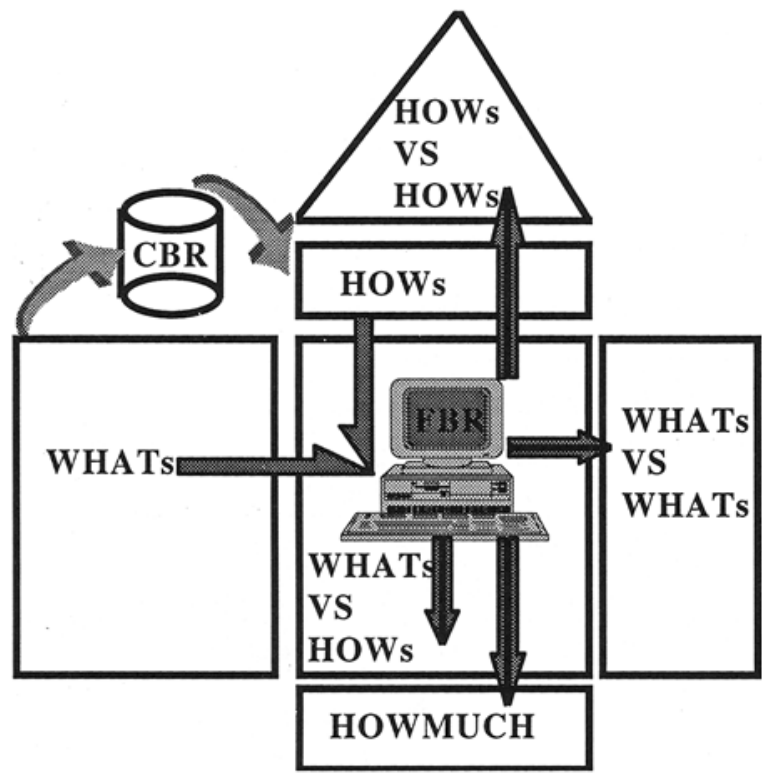

Figure 3 The new QFD-FBR methodology

Before the process of reasoning can start, the product function should be decomposed into subfunctions, which can be taken directly from the customer requirement, at the same time the generic geometric features of the product variant is to be decomposed into elementary features as shown in figure 4 for a very simple example.

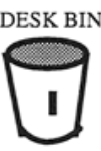

-suitable capacity

-rigid

-handy: has hand, light.

-durable

-leak-proof

-stable

-cheap

-safe: no sharp edges

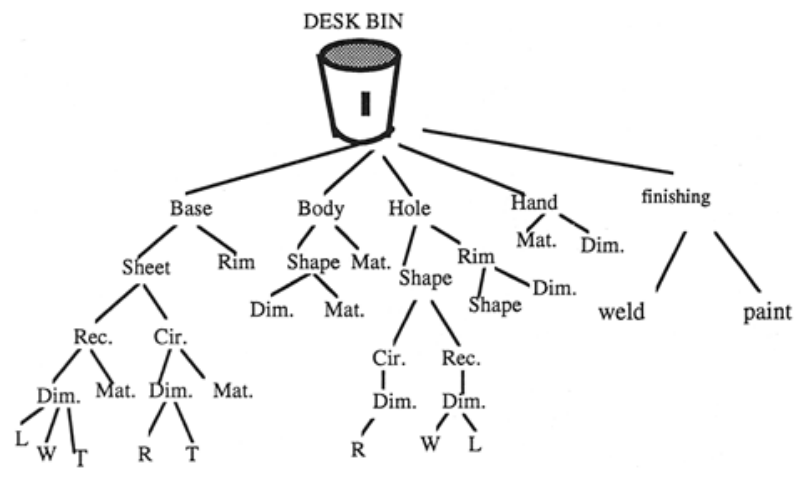

Figure 4 Decomposition of a bin's function and geometry 
The first stage of reasoning is to find the relation between these sub-functions and the elementary features, i.e. to find out what is the influence of each geometric or form feature on the functional feature of the product (see table 1). This relation can be determined and evaluated by analogy with the library of the suggested shell.

Table 1 The relation between functional and design features

\begin{tabular}{|l|l|}
\hline Functional feature & Related design features \\
\hline suitable capacity & dimensions \\
\hline rigid & material \\
\hline handy & hand,material,dimentions \\
\hline durable & material \\
\hline leak-proof & material,finishing \\
\hline stable & base:rim, dim.,body:dim. \\
\hline cheap & material,finishing \\
\hline safe & rim \\
\hline
\end{tabular}

A library of feature-based knowledge related to the generic product type supports the design parameters specification. At this stage it worth mentioning that the proposed methodology will be effective only in a variant-type design, where an increasing depth of knowledge relating to the generic product will support increasing product fitness for purpose as new variants are developed.

\section{THE HYPOTHESIS}

The overall objective of the new QFD-FBR methodology is to provide a powerful design support technique in order to achieve enhanced quality product; (and, by implication, greater customer satisfaction). The basis for the new methodology is to support the QFD by using a feature-based knowledge for the purpose of reasoning about the relation between the customer requirements and the technical specification and by achieving this goal, the following benefits of the new methodology are expected:

1. Better representation, understanding, and translation of the customer requirements.

2. The ability to adopt design changes and modifications quickly in response to the customer demands or the other stages of the product life cycle.

3. To provide an objective assessment for the relations; WHATs vs WHATs, WHATs vs HOWs, HOWs vs HOWs, and also the HOWMUCH, and the target values with a high degree of accuracy. 


\section{REFERENCES}

Adams, D. A.; Lees, B.; Irgens, C. and MacArthur, E. (1997) "Using Cases to Utilise Feedback in Quality Function Deployment" Expert Systems '97 Conference, Churchill College, Cambridge, December 15-17, 1997.

Hamza, M. (1996) The Provision Of Software Quality Advice through the Integration Of Quality Function Deployment and Case Based Reasoning Methodologies. Ph.D. thesis, University of Paisley.

Irgens, C. (1990) RA-IQSE: a system for on-line quality support for the designer of machined parts and products. Computer-Integrated Manufacturing Systems, Volume 3, Number 4; ISSN 0951-5240, Butterworth-Heinemann Ltd.

Shah, J. J. and Mantyla, M. (1995) Parametric And Feature-Based CAD/CAM Concepts, Techniques, And Applications. Wiley-Interscience Publication.

Zairi, M. (1995) Quality Function Deployment : A Modern Competitive Tool. E.F.Q.M , Technical Communication (Publishing) Ltd. 